\title{
Recoil Effects in Valence Photoemission from Simple Molecules and Clusters*
}

\author{
Ming-Hui Shang, Takashi Fujikawa, ${ }^{\dagger}$ and Nobuo Ueno \\ Graduate School of Advanced Integration Science, \\ Chiba University, 1-33 Yayoi-cho, Inage, Chiba 263-8522, Japan
}

(Received 28 December 2011; Accepted 10 April 2012; Published 27 April 2012)

\begin{abstract}
We discuss recoil effects from extended molecular orbitals in simple molecular crystals excited by high-energy X-ray photons. Because of Debye-Waller factors the interference effects between photoelectron waves emanating from different atomic sites can be neglected, and the Gelius formula is safely used, which provides us with simple expression of photoemission bands. This formula can explain the recoil energy shift and energy dependence of the intensity of each level. We apply it to the photoemission bands from acetylene and diacetylene molecular crystals in the energy range from 125 to $1200 \mathrm{eV}$. Hydrogen recoil shift is much larger than carbon, however it is hard to observe recoil effect due to the hydrogen recoil because of its quite small photoionization cross section even in this intermediate energy region. Additionally, we expect that useful information which is obtained from the energy dependence of these bands: the C $2 s$ and $2 p$ components in each molecular orbital can be estimated roughly from the energy dependence. [DOI: 10.1380/ejssnt.2012.128]
\end{abstract}

Keywords: XPS; Recoil effects; Debye-Waller factors; Franck-Condon factors

\section{INTRODUCTION}

Recent progress in high-energy photoemission from solids provide important new information [1-5], however they raises some questions about recoil effects and breakdown of electric dipole transitions. The latter problem has been studied in detail by the present authors $[6,7]$.

For the former problem, since Flynn studied the phonon broadening due to recoil phonon excitation associated with core photoemission [8], several theoretical and experimental studies have been reported. Among them, more systematic theoretical approaches have been developed by Domcke and Cederbaum for diatomic molecules [9]. Takata et al. measured high-energy-resolution C $1 s$ XPS spectra of graphite up to about $8 \mathrm{keV} \mathrm{[10].} \mathrm{With} \mathrm{increase}$ of photon energy, the $\mathrm{C} 1 s$ peak shifts to higher binding energy, which is well explained by the use of Mössbauer formula. In their theoretical approach, however, electron scatterings from surrounding atoms are completely neglected.

In our previous papers, we have studied the recoil effects on high-energy quasi-elastic scatterings and XPS spectra $[6,12,13]$. In particular the paper $[12]$ shows that simple free atom recoil energy shift $\Delta \varepsilon_{R}=-k^{2} / 2 M$, where $k^{2} / 2$ is kinetic energy of the photoelectrons, $M$ is mass of the excited atom, is accurately obtained even for all types of harmonic solids in the single-site approximation where electron scatterings from nearby atoms are completely neglected. In the derivation we have used the sum rule for the dynamical matrix for phonon spectra. The paper [13] shows the recoil effects for photoemission from extended level like molecular orbitals or solid energy bands, and also discusses the elastic scattering effects which give rise to oscillation in recoil energy shift.

Suga et al. demonstrate that the valence band recoil

* This paper was presented at the 6th International Symposium on Surface Science - Towards Nano, Bio and Green Innovation-, Tower

Hall Funabori, Tokyo, Japan, December 11-15, 2011.

†Corresponding author: tfujikawa@faculty.chiba-u.jp shifts reflects relative weights of each atomic orbital in particular of light atoms [11]. In the present paper we explicitly calculate the recoil energy shifts from extended molecular orbital in organic molecular crystals where rotational recoil effects are neglected. They are composed of only light elements like H, C and so on. For these systems even soft X-ray region (125-1200 eV) can produce the recoil shifts.

\section{BASIC PHOTOEMISSION FORMULA INCLUDING RECOIL EFFECTS}

Here we discuss the recoil effects in photoemission processes from delocalized levels, where the initial oneelectron state $\phi_{i}$ is assumed to be written as linear combination of atomic orbitals (tight-binding approximation)

$$
\phi_{i}(\mathbf{r})=\sum_{\alpha} C_{i \alpha} \chi_{\alpha}\left(\mathbf{r}-\mathbf{R}_{\alpha}\right)
$$

where $\chi_{\alpha}, \chi_{\alpha^{\prime}}, \ldots$ are atomic orbitals on the site $\mathbf{R}_{\alpha}$ and $C_{i \alpha}$ 's are the expansion coefficients. As discussed before the photoemission amplitude is now given by [7]

$$
\begin{aligned}
\left\langle\psi_{\mathbf{k}}^{-}|\Delta| \phi_{i}\right\rangle= & \sum_{\alpha} C_{i \alpha} \exp \left[-\mathbf{k} \cdot\left(\mathbf{R}_{\alpha}^{0}+\mathbf{u}_{\alpha}\right)\right] \\
& \times \sum_{L} Y_{L}(\hat{\mathbf{k}}) M_{L L_{\alpha}}+\ldots
\end{aligned}
$$

where we denote the deviation $\mathbf{u}_{\alpha}$ from the equilibrium position $\mathbf{R}_{\alpha}^{0}$ for the $\alpha$ th site. The atomic excitation amplitude $M_{L L_{\alpha}}$ describes the photoexcitation from the atomic orbital $\chi_{\alpha}$ with $L_{\alpha}=\left(l_{\alpha}, m_{\alpha}\right)$ to the photoelectron state with angular momentum $L=(l, m)$. The first term describes the photoemission processes without suffering elastic scatterings from surrounding atoms. Within this approximation, we obtain the photoemission intensity formula

$$
I_{0}(\mathbf{k})_{i}=I_{0}(\mathbf{k})_{i}^{1}+I_{0}(\mathbf{k})_{i}^{2}
$$


where $I_{0}(\mathbf{k})_{i}^{1}$ and $I_{0}(\mathbf{k})_{i}^{2}$ are the one- and two-center terms. The former is now written

$$
\begin{aligned}
& I_{0}(\mathbf{k})_{i}^{1} \propto \sum_{\alpha \alpha^{\prime}} C_{i \alpha}^{*} C_{i \alpha^{\prime}} \int \frac{d t}{2 \pi} \exp (i \varepsilon t) \\
& \times\left\langle\exp \left(-i \hat{A}_{\alpha}(t)\right) \exp \left(i H_{v} t\right) \exp \left(-i H_{v}^{*} t\right) \exp \left(i A_{\alpha}\right)\right\rangle \\
& \times \sum_{L L^{\prime}} Y_{L}^{*}(\hat{\mathbf{k}}) Y_{L^{\prime}}(\hat{\mathbf{k}}) M_{L L_{\alpha}}^{*} M_{L^{\prime} L_{\alpha^{\prime}}} \\
& \hat{A}_{\alpha}(t)=\exp \left(i H_{v} t\right) A_{\alpha} \exp \left(-i H_{v} t\right)
\end{aligned}
$$

We assume that linear approximation works well even for valence photoemission

$$
H_{v}^{*}=H_{v}+\sum_{s}\left(\lambda_{s} b_{s}+\lambda_{s}^{*} b_{s}^{\dagger}\right)
$$

where $b_{s}\left(b_{s}^{\dagger}\right)$ is the phonon annihilation (creation) operator for the mode $s$. The one-center term is thus given by [13]

$$
\begin{aligned}
I_{0}(\mathbf{k})_{i}^{1} & =\sum_{\alpha \alpha^{\prime}} C_{i \alpha}^{*} C_{i \alpha^{\prime}} \int_{-\infty}^{\infty} \frac{d t}{2 \pi} \exp \left[i \varepsilon t+\gamma(t)-F_{\alpha}(t)-G_{\alpha}(t)\right] \\
& \times \sum_{L L^{\prime}} Y_{L}^{*}(\hat{\mathbf{k}}) Y_{L^{\prime}}(\hat{\mathbf{k}}) M_{L L_{\alpha}}^{*} M_{L^{\prime} L_{\alpha^{\prime}}}
\end{aligned}
$$

In Eq. (6) the term $\gamma(t)$ is related to the Franck-Condon effects, $F_{\alpha}(t)$ to the recoil effects, and $G_{\alpha}(t)$ to the interference between them. The explicit formulas of them are shown in Ref. [13]. When we neglect the interference terms, we obtain the energy shift as for the core excitation $[12]$,

$$
\Delta \varepsilon_{\alpha}=-k^{2} /\left(2 M_{\alpha}\right)
$$

The previous analyses show that the off-diagonal terms $\left(\alpha \neq \alpha^{\prime}\right)$ in the sum (6) can be neglected [14]. Finally a very simple formula is obtained after we average over $\hat{\mathbf{k}}$,

$$
I_{0}(\omega)_{i}^{1} \propto \sum_{\alpha}\left|C_{i \alpha}\right|^{2} \sigma_{\alpha} \delta\left(\omega-\varepsilon_{k}-\varepsilon_{i}-\Delta \varepsilon_{\alpha}\right)
$$

where $\varepsilon_{k}$ is the kinetic energy of photoelectron, $\varepsilon_{i}$ is the binding energy of the $i$-th orbital, and $\sigma_{\alpha}$ is the partial cross section from $\alpha$ th atomic orbital

$$
\sigma_{\alpha} \propto \sum_{L}\left|M_{L L_{\alpha}}\right|^{2}
$$

So far we have neglected higher terms of $t$ than the linear term in $\gamma(t)-F_{\alpha}(t)-G_{\alpha}(t)$ in Eq. (6). Now the terms up to the second power of $t$ is taken into account to discuss the peak width. The integrals over $t$ are analytically carried out, which yields [13]

$$
\begin{aligned}
& I_{0}(\omega)_{i}^{1} \propto \sum_{\alpha}\left(\sum_{\alpha^{\prime}}\left|C_{i \alpha^{\prime}}\right|^{2} \sigma_{\alpha^{\prime}}\right) g_{\alpha}^{i}(\omega) \\
& g_{\alpha}^{i}(\omega)=\frac{1}{\sqrt{2 \pi F_{\alpha}}} \exp \left[-\frac{\left(\omega-\varepsilon_{k}-E_{i}-\Delta \varepsilon_{\alpha}\right)^{2}}{2 F_{\alpha}}\right]
\end{aligned}
$$

where the width parameter $F_{\alpha}$ is calculated by

$$
F_{\alpha}=k^{2} \int_{0}^{\infty} d \omega \omega^{2} a_{\alpha}(\omega)[2 n(\omega)+1] .
$$

The sum of $\alpha^{\prime}$ in Eq. (10) runs over atomic orbitals on the site $\alpha$. The projected phonon spectral function on the site $\alpha, a_{\alpha}(\omega)$ is given in terms of eigenvectors of the phonon dynamical matrix which is linear function of $k$ [13]. The width parameters depend on temperature which is slowly increasing function of the temperature $T$. At $300 \mathrm{~K}$ it is about $0.1 \mathrm{eV}$ for graphite [12].

The two-center term is similarly written as [13]

$$
\begin{aligned}
I_{0}(\mathbf{k})_{\mathbf{i}}^{2} & =\sum_{\alpha \beta} C_{i \alpha} C_{i \beta}^{*} \int_{-\infty}^{\infty} \frac{d t}{2 \pi} \exp \left[i \varepsilon t+\gamma(t)-F_{\alpha \beta}(t)-G_{\alpha \beta}(t)\right] \\
& \times \exp \left[i \mathbf{k} \cdot\left(\mathbf{R}_{\alpha}^{0}-\mathbf{R}_{\beta}^{0}\right)\right] \sum_{L L^{\prime}} Y_{L}^{*}(\hat{\mathbf{k}}) Y_{L^{\prime}}(\hat{\mathbf{k}}) M_{L L_{\beta}}^{*} M_{L^{\prime} L_{\alpha}},
\end{aligned}
$$

where the two-center "recoil factor" $F_{\alpha \beta}(t)$ includes the time-independent Debye-Waller factor

$$
\begin{aligned}
F_{\alpha \beta}(t) & =k^{2}\left\langle\Delta_{\alpha \beta}^{2}\right\rangle / 2+\tilde{F}_{\alpha \beta}(t) \\
\Delta_{\alpha \beta} & =\hat{\mathbf{k}} \cdot\left(\mathbf{u}_{\alpha}-\mathbf{u}_{\beta}\right)
\end{aligned}
$$

The thermal damping factor $\exp \left(-k^{2}\left\langle\Delta_{\alpha \beta}^{2}\right\rangle / 2\right)$ amounts to $\approx 0.2$ when $\varepsilon_{k}=5 \mathrm{keV}$ and $\left\langle\Delta_{\alpha \beta}^{2}\right\rangle / 2=10^{-2} a . u .^{2}$ as in typical condensed systems. We thus can neglect the twocenter term $I_{0}(\mathbf{k})_{i}^{2}$ compared with the one-center term $I_{0}(\mathbf{k})_{i}^{1}$ in this high-energy region. 


\section{ACETYLENE}
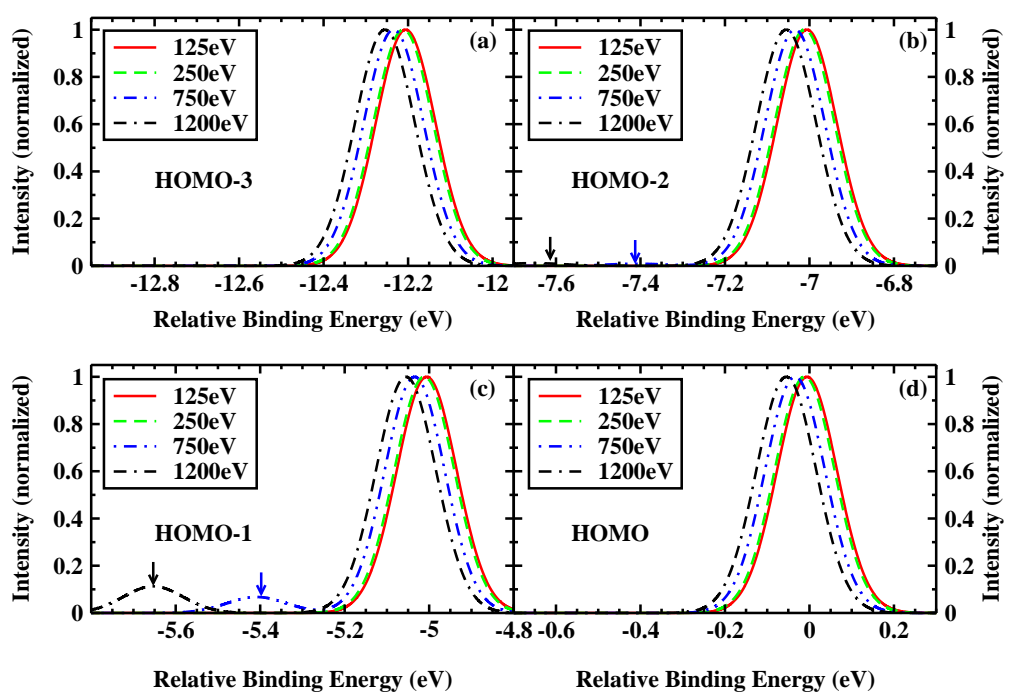

FIG. 1: Calculated energy dependence of the photoemission bands for four molecular orbitals in acetylene molecular crystals. The energies indicated in the figure show the photoelectron kinetic energies. The arrows show the shoulder due to hydrogen recoil.

DIACETYLENE
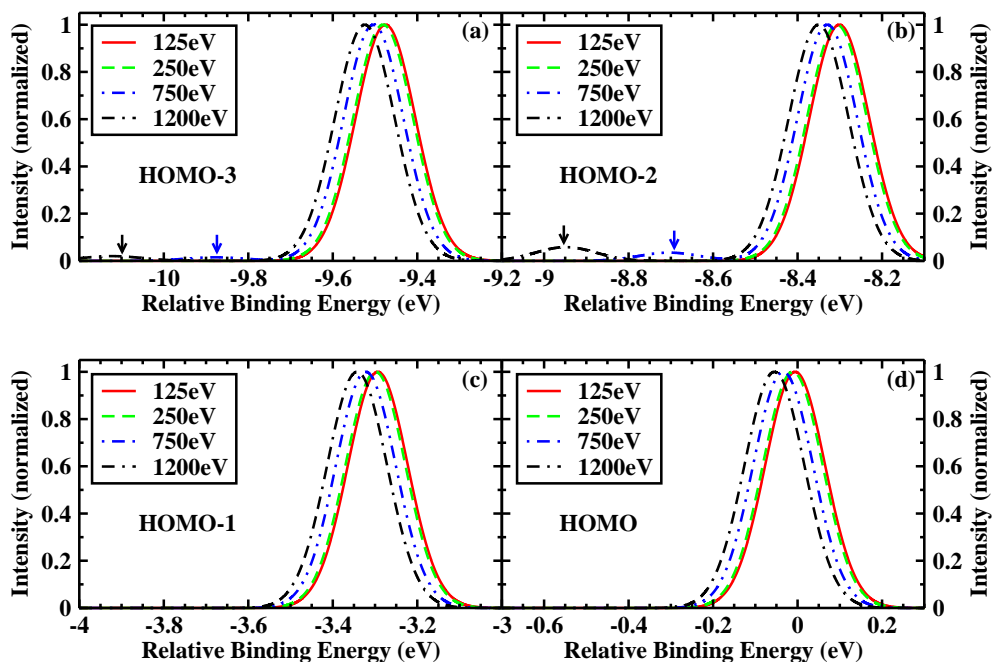

FIG. 2: Calculated energy dependence of the photoemission bands for four molecular orbitals in diacetylene molecular crystals. The energies indicated in the figure show the photoelectron kinetic energies. The arrows show the shoulder due to hydrogen recoil.

\section{RESULTS AND DISCUSSION}

As discussed above the recoil shift is remarkable for light elements like $\mathrm{H}$ and $\mathrm{C}$, but their photoionization cross sections rapidly decrease as a function of the kinetic energy. We thus calculate the recoil effects on the photoemission bands from extended molecular orbitals in simple organic molecules like acetylene and diacetylene. At room temperature they are free molecules: We rather consider the photoemission from their crystals. In this case rotational recoil can be neglected [9], and fine vibrational structures should be smeared out. The theoretical formulas shown in the previous section thus can be applied.

The classical recoil energy shifts for free $\mathrm{H}$ and $\mathrm{C}$ atoms are simply calculated by use of Eq. (7), which are shown
TABLE I: Recoil energy shift in eV ejected from $\mathrm{C}$ and $\mathrm{H}$ atoms for different photoelectron energies.

\begin{tabular}{ccccc}
\hline \hline$\varepsilon_{k}(\mathrm{eV})$ & 125 & 250 & 750 & 1200 \\
\hline $\mathrm{H}$ & 0.068 & 0.136 & 0.408 & 0.653 \\
$\mathrm{C}$ & 0.0057 & 0.0114 & 0.0343 & 0.0549 \\
\hline \hline
\end{tabular}

in Table I. Even at $750 \mathrm{eV}$, the recoil shift for $\mathrm{H}$ atom amounts to $0.41 \mathrm{eV}$ which can be well resolved experimentally.

In order to calculate the recoil spectra, we now apply the Gaussian code [16] to calculate the population of $\alpha^{\prime}$ atomic orbital in each molecular orbital and the results are summarized in Table II. Figure 1 shows the energy 
TABLE II: The population of $\mathrm{H} 1 s, \mathrm{C} 2 s$ and $\mathrm{C} 2 p$ in each molecular orbital of acetylene and diacetylene.

\begin{tabular}{|c|c|c|c|c|c|c|}
\hline \multirow[b]{2}{*}{ molecular orbital } & \multicolumn{3}{|c|}{ acetylene } & \multicolumn{3}{|c|}{ diacetylene } \\
\hline & $\mathrm{H} 1 s$ & $\mathrm{C} 2 s$ & C $2 p$ & $\mathrm{H} 1 s$ & $\mathrm{C} 2 s$ & $\mathrm{C} 2 p$ \\
\hline HOMO & 0.0000 & 0.0000 & 1.0000 & 0.0000 & 0.0000 & 1.0000 \\
\hline HOMO-1 & 0.3745 & 0.0148 & 0.6108 & 0.0000 & 0.0000 & 1.0000 \\
\hline HOMO-2 & 0.3607 & 0.3548 & 0.2372 & 0.2342 & 0.0170 & 0.7488 \\
\hline HOMO-3 & 0.0468 & 0.7364 & 0.0953 & 0.3490 & 0.1882 & 0.4392 \\
\hline
\end{tabular}

TABLE III: The relative photoionization cross section of $\mathrm{H} 1 s, \mathrm{C} 2 s$ and $\mathrm{C} 2 p$ normalized to that of $\mathrm{C} 2 s$ at each kinetic energy. Multi-configuration interaction atomic wave functions are used.

\begin{tabular}{cccr}
\hline \hline$\varepsilon_{k}(\mathrm{eV})$ & $\mathrm{H} 1 s$ & $\mathrm{C} 2 s$ & $\mathrm{C} 2 p$ \\
\hline 125 & $2.30 \mathrm{E}-04$ & $1.00 \mathrm{E}+00$ & $1.52 \mathrm{E}+00$ \\
\hline 250 & $2.40 \mathrm{E}-03$ & $1.00 \mathrm{E}+00$ & $5.70 \mathrm{E}-01$ \\
\hline 750 & $2.00 \mathrm{E}-02$ & $1.00 \mathrm{E}+00$ & $1.57 \mathrm{E}-01$ \\
\hline 1200 & $3.07 \mathrm{E}-02$ & $1.00 \mathrm{E}+00$ & $1.42 \mathrm{E}-01$ \\
\hline \hline
\end{tabular}

dependence of the photoemission bands from four molecular orbitals of an acetylene molecular crystal for different photoelectron kinetic energies in the range 125-1200 $\mathrm{eV}$. The peak heights are normalized to be the same for these different photon energies: They should get weaker with photon energy. The energy dependence of photoionization cross section for each atomic orbital is shown in Table III. We should note that that for C $2 p$ rapidly decreases with the photoelectron kinetic energy $\varepsilon_{k}$. The highest occupied orbital (HOMO) is purely composed of $\mathrm{C} 2 p$ orbitals which give rise to the recoil shift about 30 meV for $\varepsilon_{k}=750 \mathrm{eV}$ and $\sim 50 \mathrm{meV}$ for $\varepsilon_{k}=1200 \mathrm{eV}$. The deeper orbitals (HOMO-1 $\sim$ HOMO-3) also show nearly the same behaviors as the HOMO, but HOMO-1 $\left(\sigma_{g}\right)$ yields a prominent shoulder due to the hydrogen recoil at 750 and $1200 \mathrm{eV}$. In that molecular orbital the population of $\mathrm{H} 1 s$ is quite large ( $\sim 37$ percent). The HOMO-2 $\left(\sigma_{u}\right)$ also shows a very small shoulder, though the $\mathrm{H} 1 s$ population is nearly the same ( $\sim 36$ percent). In HOMO- $1, \mathrm{C} 2 p$ is dominant; $\sigma(H 1 \mathrm{~s}) / \sigma(C 2 \mathrm{p})$ is not so small whereas the ratio $\sigma(H 1 \mathrm{~s}) / \sigma(C 2 \mathrm{~s})$ is quite small. Referring to Eq. (10), we could expect weak shoulder due to hydrogen large recoil shift as far as $\mathrm{H} 1 s$ photoionization cross section is

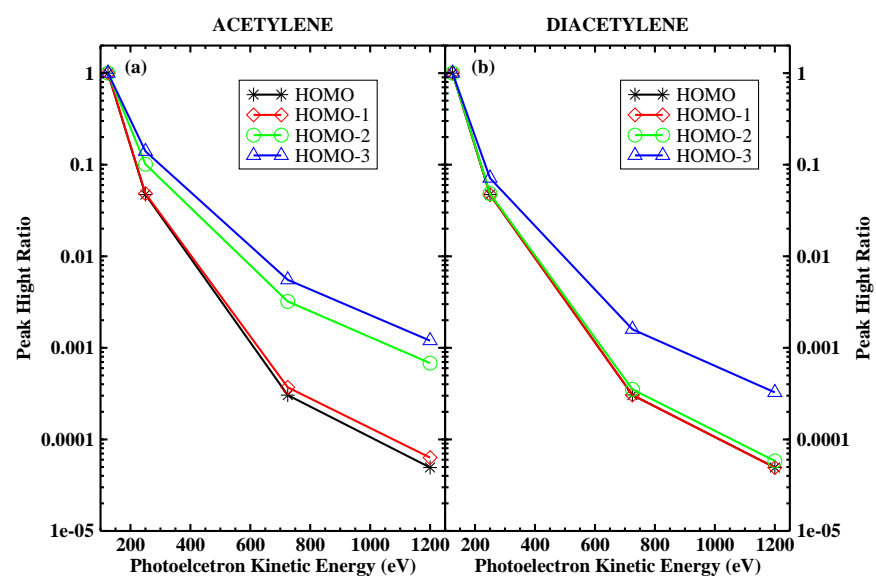

FIG. 3: Energy dependence of the peak height of each photoemission band of an acetylene (a) and a diacetylene (b) crystal, which reflects the $\mathrm{H} 1 s, \mathrm{C} 2 s$ and $2 p$ components in each molecular orbital. not negligibly small compared with those for $\mathrm{C} 2 s$ and $2 p$ orbitals (see Table III). For HOMO-1 and HOMO-2, considerable weight is contributed by $\mathrm{H} 1 s$ (see Table II), thus in both Figs. 1 (b) and (c) we can obtain the small peaks at the binding energy $\sim-0.364 \mathrm{eV}$ and $\sim-0.605$ $\mathrm{eV}$ from the main peak, especially for HOMO-1. And the energy shift is exactly the difference between recoil energy for $\mathrm{H}$ and $\mathrm{C}$ by $750 \mathrm{eV}$ and $1200 \mathrm{eV}$, which can be directly estimated from Table I.

Likewise, such kind behaviors are also obtained in the energy dependent photoemission spectra from diacetylene as shown in Fig. 2. In this system the recoil shoulder is observed for HOMO-2: We can explain the reason refereing to Table II. Namely, the top 2 MOs are $\pi$ orbital purely composed of C $2 p$. For diacetylene crystal, HOMO-3 has large components of C $2 s$ orbital (see also Table II). And small peaks appear in lower energy region with respect to the main peaks under the joint action of noneligible photoionization cross section and considerable component of $\mathrm{H} 1 s$.

What useful information can we extract from the energy dependent photoemission bands in these energy region? Figure 3 shows the energy dependence of the peak height (see Eq. (10)) of each photoemission band of acetylene and diacetylene crystal, which mainly reflects only $\mathrm{C} 2 s$ and $2 p$ components. When it decays rapidly with photon energy, the $\mathrm{C} 2 p$ components dominantly contributes to the occupied molecular orbital. This figure clearly shows that two deeper $\sigma$ orbitals (HOMO-3 and HOMO-2) have larger components of $\mathrm{C} 2 s$ orbital than the $\pi$ orbital (HOMO) in acetylene. That is also the case for HOMO-3 in diacetylene.

\section{CONCLUDING REMARKS}

We discuss recoil effects from extended molecular orbitals in simple molecular crystals excited by high-energy X-ray photons. Because of Debye-Waller factors the interference effects between photoelectron waves emanating from different atomic sites can be neglected, and the Gelius formula is safely used, which provides us with simple expression of photoemission bands. This formula can 
explain the recoil energy shift and energy dependence of the intensity of each level. We apply it to the photoemission bands from acetylene and diacetylene molecular crystals in the energy range from 125 to $1200 \mathrm{eV}$. Hydrogen recoil shift is much larger than carbon, however it is hard to observe the recoil effect due to the hydrogen recoil because of its quite small photoionization cross section even in this intermediate energy region. When the $\mathrm{H} 1 s$ population is large in that molecular orbital, we can expect the recoil shoulder due to large recoil shift of hydrogen. We expect that useful information is obtained from the energy dependence of these bands: The C $2 s$ and $2 p$ components in each molecular orbital can be estimated from the energy dependence.

Hydrogen gives rise to the largest recoil shift, but has smallest photoionization cross section in the high-energy region considered here. In contrast to the photoemission quasi-elastic backscattering experiments can provide us with recoil peak shift because of still observable elastic scattering intensity [15]. When these molecules are composed of heavy elements like $\mathrm{Si}, \mathrm{S}, \mathrm{Cl}$ in addition to $\mathrm{H}$ and $\mathrm{C}$, we can obtain which MO's have large contribution from these atoms because they should show smaller recoil shifts. Further detailed studies will be discussed in forthcoming paper.

\section{Acknowledgments}

The authors are grateful to useful comments on this manuscript by H. Shinotsuka, M. Kazama, Y. Ohori, and M. Nagaosa. This work is supported by the Global COE Program (Advanced School for Organic Electronics) Chiba University.
[1] A. Sekiyama and S. Suga, Physica B 312-313, 634 (2002).

[2] A. Sekiyama and S. Suga, J. Electron Spectrosc. Relat. Phenom. 137-140, 681 (2004).

[3] A. Yamasaki, A. Sekiyama, S. Imada, M. Tsunekawa, C. Dallera, L. Braicovich, T. Lee, A. Ochiai, and S. Suga, J. Phys. Soc. Jpn. 74, 2538 (2005).

[4] S. Suga, Appl. Phys. A 92, 479 (2008).

[5] S. Suga and A. Sekiyama, J. Electron Spectrosc. Relat Phenom. 181, 48 (2010).

[6] R. Suzuki, H. Arai, H. Shinotsuka, and T. Fujikawa, e-J. Surf. Sci. Nanotech. 3, 373 (2005).

[7] T. Fujikawa, R. Suzuki, H. Arai, H. Shinotsuaka, and L. Köver, J. Electron Spectrosc. Relat. Phenom. 159, 14 (2007).

[8] C. P. Flynn, Phys. Rev. Lett. 37, 1445 (1976).

[9] W. Domcke and L. S. Cederbaum, J. Electron Spectrosc. Relat. Phenom. 13, 161 (1978).

[10] Y. Takata, Y. Kayanuma, M. Yabashi, M. Tamasaka, Y. Nishino, D. Miwa, Y. Harada, S. Shin, S. Sanaka, E. Ikenaga, K. Kobayashi, Y. Senba, H. Obashi, and T. Ishikawa, Phys. Rev. B 75, 233404 (2007) .

[11] S. Suga, A. Sekiyama, H. Fujiwaea, Y. Nakatsu, T. Miyamachi, S. Imada, P. Baltzer, S. Niitaka, H. Takagi, K. Yoshimura, M. Yabashi, K. Tamasaku, A. Higaashiyama, and T. Ishikawa, New J. Phys. 11, 073025 (2009).

[12] T. Fujikawa, R. Suzuki, and L. Köver, J. Electron Spectrosc. Relat. Phenom. 151, 170 (2006).

[13] T. Fujikawa, H. Arai, R. Suzuki, H. Shinotsuaka, L.
Köver, and N. Ueno, J. Electron Spectrosc. Relat. Phenom. 162, 146 (2008).

[14] T. Fujikawa, J. Phys. Soc. Jpn. 50, 1321 (1981).

[15] A. Sulyok, G. Gergely, M. Menyhaqrd, J. Toth, D. Varga, L. Köver, Z. Berenyi, B. Lesiak, and A. Kosinki, Vacuum 63, 371 (2001).

[16] Gaussian 03, Revision C.02, M. J. Frisch, G. W. Trucks, H. B. Schlegel, G. E. Scuseria, M. A. Robb, J. R. Cheeseman, J. A. Montgomery, Jr., T. Vreven, K. N. Kudin, J. C. Burant, J. M. Millam, S. S. Iyengar, J. Tomasi, V. Barone, B. Mennucci, M. Cossi, G. Scalmani, N. Rega, G. A. Petersson, H. Nakatsuji, M. Hada, M. Ehara, K. Toyota, R. Fukuda, J. Hasegawa, M. Ishida, T. Nakajima, Y. Honda, O. Kitao, H. Nakai, M. Klene, X. Li, J. E. Knox, H. P. Hratchian, J. B. Cross, C. Adamo, J. Jaramillo, R. Gomperts, R. E. Stratmann, O. Yazyev, A. J. Austin, R. Cammi, C. Pomelli, J. W. Ochterski, P. Y. Ayala, K. Morokuma, G. A. Voth, P. Salvador, J. J. Dannenberg, V. G. Zakrzewski, S. Dapprich, A. D. Daniels, M. C. Strain, O. Farkas, D. K. Malick, A. D. Rabuck, K. Raghavachari, J. B. Foresman, J. V. Ortiz, Q. Cui, A. G. Baboul, S. Clifford, J. Cioslowski, B. B. Stefanov, G. Liu, A. Liashenko, P. Piskorz, I. Komaromi, R. L. Martin, D. J. Fox, T. Keith, M. A. Al-Laham, C. Y. Peng, A. Nanayakkara, M. Challacombe, P. M. W. Gill, B. Johnson, W. Chen, M. W. Wong, C. Gonzalez, and J. A. Pople, Gaussian, Inc., Wallingford CT (2004). 